\title{
The SEED Diagonal Concept Cleft Care Centre in a Covid-19 Affected World: Safety, Economics, and Education Delivery for Patients and Trainee Cleft Surgeons
}

\author{
Bona Lotha ${ }^{1 *}$ and Zeinalddin $\mathbf{M}^{2}$ \\ ${ }^{1}$ Craniofacial Cleft Surgeon, Yemen Global Smiles, Sanaa, Yemen \\ ${ }^{2}$ Craniofacial Orthodontist, Mohammad Orthodontic Center, Senior Lecturer at \\ Oman Dental College, Muscat, Sultanate of Oman \\ *Corresponding Author: Bona Lotha, Craniofacial Cleft Surgeon, Yemen Global \\ Smiles, Sanaa, Yemen.
}

Received: February 22, 2021

Published: March 15, 2021

C) All rights are reserved by Bona Lotha and

Zeinalddin M.

\section{Abstract}

Since Covid-19, elective consultations have been mostly replaced by online consultations, telemedicine, WhatsApp consults, and other forms of remote patient management. Unless proved otherwise, all patients requiring surgery should be considered potentially infected; healthcare staff in operating theatres must always use PPE regardless of the COVID status of a patient. Developing countries with struggling economies can ill afford to give top priority to cleft populations in need. Cleft children mostly live with their deformities for life. With Covid 19, the problem has become even worse, making it very difficult for overburdened governments to care for elective, humanitarian surgeries.

The concept of the SEED (Safety, Economics, and Education Delivery) diagonal cleft center was designed in Yemen and implemented across the entire country since the late 1990s where safety, economics and education delivery was practiced across different target zones, including government and private hospitals in the country. This enabled us to reach underprivileged populations across the nation, in both urban and rural areas at a minimal cost.

The programs find synergy between the immediate advantages of vertical inputs (sponsor groups) and the long-term benefits of horizontal aims (local beneficiary hospital unit), ultimately increasing access and enriching capacity of surgical services across the country, making it one of the most cost-effective ways of reaching underprivileged cleft patient groups. The concept improves better social understanding in the community as cleft patients integrate into normal society and are able to contribute to the system to enhance quality of life for all.

Surgeons involved in cleft care can provide a safe and sustainable smile mission in poorer regions using their skills, as well as proper financial support systems for the programmes.

Keywords: Cleft Training; Cleft Protocol; Health Equity; Safety Protocol in Covid-19; Smile Outreach

\section{Introduction}

Since Covid-19, elective consultations have been mostly replaced by online consultations, telemedicine, WhatsApp consults, and other forms of remote patient management. Unless proved otherwise, all patients requiring surgery should be considered potentially infected; healthcare staff in operating theatres must always use PPE regardless of the COVID status of a patient [1].

Developing countries with struggling economies are generally resource poor and can ill afford to give top priority to cleft 
populations in need. Poorly paid surgeons in these regions often have little incentive or interest to prioritize cleft care. The cleft children live with their deformities for life or are left at the mercy of safari smile surgeons [2].

The concept of the SEED diagonal cleft center was designed in Yemen and implemented across the country since the late 1990s where safety, economics and education delivery was practiced across different government and private hospitals in the country. This enabled us to reach underprivileged populations in both urban and rural areas. Several years later, similar models were proposed by several international groups to reach out to underprivileged populations [3].

The SEED diagonal concept programs find synergy between the immediate advantages of vertical inputs (sponsor groups) and the long-term benefits of horizontal aims (local beneficiary hospital unit), ultimately increasing access and enriching capacity of surgical services. Health inequalities are faced by many governments across the world with underprivileged populations at greatest risk of neglect due to failure of government policies [4].

In 2010 January, the Smile Train NYC sponsored all the cleft lip and palate children under our programmes across the entire nation. An extra 1490 cleft lip and palate patients were helped till March 2015. In 2020, during the Covid -19 global crisis, a global online smile training network Yemen Global Smiles was started by the craniofacial cleft founder, well-wishers and a compassionate craniofacial orthodontist Dr. Mohammad Zeinalddin from Muscat, Oman who kindly offered to sponsor all the craniofacial cleft scientific research papers for the benefit of younger cleft trainees.

The majority of the world's population with clefts come from the developing world. The need of the hour is to market the affordable, applicable, reproducible take-home message for cleft trainees so that more underprivileged patients can receive quality cleft care. A main cleft lip and palate center is used for training target regions where local surgeons can reproduce the same in an efficient and cost effective manner [5].

Trainee cleft surgeons in developing countries want to go beyond textbook or safari cleft surgery teaching to real experience in standardized cleft lip and palate surgery techniques-the SEED Diagonal Concept Cleft Centre may provide some of the facilities by well-seasoned primary craniofacial cleft specialists with a proven track record.

\section{Purpose of the Study}

The purpose of this article is to stress the importance of basic safety during Covid 19 and also suggest ways one could run a sustainable smile enterprise for all, taking advantage of skills as well as a financial support structure.

\section{Materials and Methods}

While prioritizing cleft care delivery, we would like to suggest the three important areas of safety, economics and education. The learning curve for cleft surgery, patient safety in Covid-19 will be long since all of us are new to this crisis. Hospitals are rapidly becoming hot zones and it makes sense to postpone elective surgeries. Aerosol generating procedures (AGPs) are associated with an increased risk to the health care providers as well as patients [6].

All AGPs of the aero-digestive tract (nasopharyngeal, oropharyngeal, tracheal, lung) were considered high risk given the known viral load in these areas and potential for aerosolization, consistent with previous experience with SARS.

The C-19 SEED Diagonal Concept Cleft Centre should meet the following requirements:

1. PPE requirement for the entire team -droplet PPE (gown, gloves, eye protection)

2. N 95 respirator masks for the medical team

3. Institutional access to laboratory testing - any patient could be infected with COVID-19 unless proven otherwise [7].

In addition, the UK Royal Surgical Colleges advise that any patients for prioritized, planned, surgery must have been asymptomatic for 7 days, self-isolated for 14 days and then have a negative swab within $48 \mathrm{~h}$ prior to planned admission as well as to be assessed for COVID-19 [8].

The World Health Organization Surgical Safety Checklist is used for all sponsored surgeries. Safety initiatives for cleft care demonstrate beneficial effects extending to the target partner hospitals [9]. 


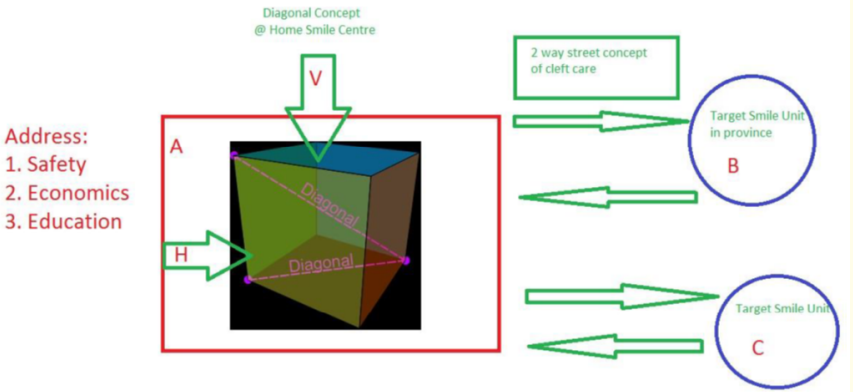

Figure 1: The SEED diagonal concept cleft care centre (courtesy L Bona) a diagonal approach harnesses the power of horizontal aims and vertical inputs.

The main goal of the vertical and horizontal care systems is to provide sustainable, local care delivery systems. Seeding of specialist medical teams in local hospitals is a useful means to achieving better outcomes [10].

Public education on clefts through social media is equally important to remove misconceptions about cleft deformities and acceptance as normal individuals into society [11].

Cleft surgeon education: the 2 way street model

Transport to main smile center: all complicated cases (temporary measure)

Provide trainings on a regular basis to cleft trainees and other staff-social workers, nurses.

Take the operation theatre demos to patient province hospitalsPatients too far away and poor to afford travel.

Building network across region with public, medical teams, governments.

Our proposed protocol: Cleft surgeons have different preferences for their treatment protocols but most of the procedures followed are similar standardized techniques with some variations according to team preference.

\section{Our proposed 2021 cleft protocol from birth till maturity}

1. Pre surgical care: Naso Alveolar molding in the first week [12].
2. Unilateral primary lip and nose: 3 months, C flap cheiloplasty and variations [13].

3. Bilateral primary lip: 3 months, Using Mulliken's five principles of symmetrical repair with nuanced modifications [14].

4. Primary cleft palate: 9 - 12 months, Modifications and variations of the $\mathrm{Z}$ flaps [15].

5. VPI surgery: Furlows is shown to be superior to other methods like midline flaps and IVVP; buccal flaps are useful for some wide defects; a good primary repair prevents fistulae [16].

6. The growing skeleton: Alveolar bone graft (ABG) at age 9 or 10 with scarpa fascia plug [17].

7. The mature skeleton: Part I Orthognathic Surgery (OGS) is needed in 30 - 40\% of cleft patients. We prefer Surgery First Le Fort 1 and Bilateral Sagittal Split Osteotomy (LF1 and BSSO) [18].

8. Mature cleft rhinoplasty [19]: Correction of the cleft nasal deformity: from infancy to maturity.

How the seed diagonal concept cleft centre works

Visiting trainees could be mentored and instructed in these areas by local providers with expertise in local systems and caredelivery challenges.

Faculty, learning clinical skills through intraoperative teaching sessions, formal lectures, and skills based workshops.

This approach would benefit local trainees and, ultimately, the patients they will serve in their home communities.

Education for: General surgeons/ENT/OMS/PLASTIC/pediatric and other surgical subspecialists.

Capacity building in resource limited settings; greater numbers of well-trained surgeons, scrub technicians, nurses and anesthesiologists would improve surgical care for all patients.

\section{Economics: Diagonal financing for the work}

Patient travel costs, the most commonly reported barrier to care for CLP, also represents one of the largest out-of-pocket expenditures encountered when seeking healthcare.

Cleft surgeons/medical teams need incentives to work locally. 
Finances for cleft care programmes: Vertical financing for disease specific (clefts) can be provided by donors, government funded programmes. Horizontal financing needs, aimed at improving cleft center are often met by local institutions (Corporate sector, hospital, business). External sources: such as donations from non-governmental organizations, transfers from donor agencies and loans from international banks, also contribute significantly in some countries, especially low-and middle-income countries (LMIC) [20].

\section{The Yemen example}

In order to meet the growing needs of cleft and other plastic surgery patients in Yemen, a small team of volunteers worked on the following to establish an International charity NGO for the patients:

- Formulating a Governing document: This was very kindly prepared by HMA Mike Gifford, UK Res and his team of expat Trustees using some helpful guidelines from the London Charities Commission. (London Charities Commission: Charity Guidelines www.gov.uk/government/organizations/ charity-commision).

The project was launched for furthering the following objectives:

1. To provide free or low cost medical services

2. To provide training in cleft lip and palate surgery.

\section{Delegation}

The first trustees and their respective terms of office are listed below:

1. Michael John Gifford, London one year

2. Patricia Anne Gifford, one year

3. Colin Kramer, Five years GM, DNO Yemen Sana'a Republic of Yemen

4. Andre Armand Jean Louis Lamy, two years Total E\&P Yemen

5. S Vilvanathan, Five years Finance Controller, DNO Yemen

6. Stan Hazell, Three years Bristol

7. Jill Hazell, Three years Bristol.

Funding for the projects through locally based expat companies and other donors; the main donors were DNO Norway and France's Total E\&P Oil Company along with other donors.
Forming a medical support team that would address the cleft and other plastic surgery problems by a locally based team.

Patient database system: The Govt and private hospitals have their own database system in Arabic.
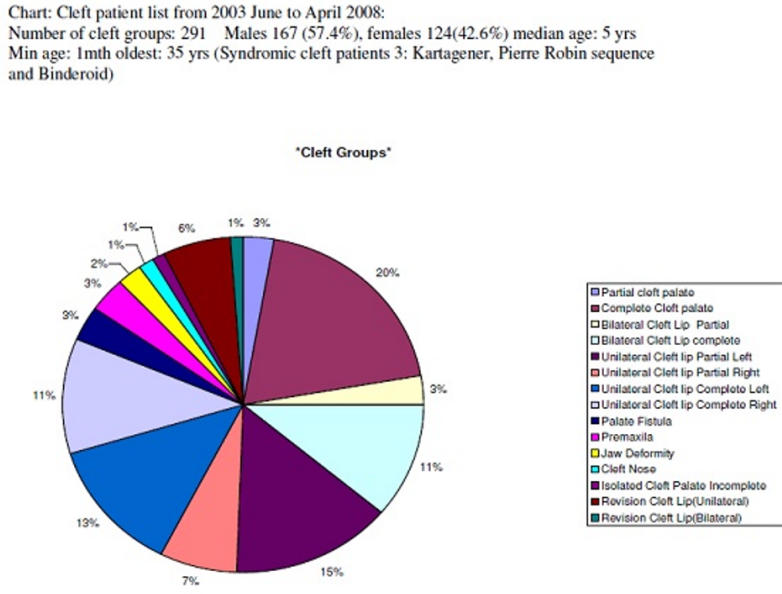

Figure 2: Cleft Patient from 2003 - 2008 (Courtesy Dr. Bona Lotha, Yemen Smile Founder).

\section{Results and Observations}

The concept was found to be effective in addressing major cleft problems in poor resource nations where cleft patients are at a huge disadvantage in society.

The most important requirements for a smile cell are:

1. A local hospital unit with a trained surgeon, anesthetist, nurses and medical technicians.

2. Basic medical equipment for the surgery which include: anesthesia machine, monitor, cautery machine, sterilizers, OT table, suction units, plastic surgical instruments and adequate lighting.

From 2003 June till March 2008 over 550 patients were able to attend our clinics and by March 2009 over 544 extra patients were successfully treated for clefts and other plastic surgery problems. By 2015, sponsorship of 1490 clefts were granted by the Smile Train NYC. The complete report of our numbers and detailed 
procedures is being prepared in the form of several reports. The percentage of secondary lip and palate revisions was reasonably low at about 2 - 3 percent. The palate fistula was mostly at the junction and did not require treatment in over 95\% cases, except for a small number, less than $2 \%$.

There are important lessons we have learnt from our experience and some of the improvements needed include:

1. A good team of craniofacial surgeons and orthodontists to handle cleft related problems over the long term.

2. A diagonal concept of cleft care with synergy between the vertical (funding) system and horizontal (health delivery) team.

3. Regular updates and training for medical personnel in the latest approaches to cleft care.

Funding for the projects through locally based expat companies and other donors (Smile Train., et al).

An approximate budget is planned for the years programme and the government, well-wishers, donors, corporate sector make pledges of regular support to enable the horizontal input team to carry on the work.

Forming a local medical support team [21]

Training local cleft surgeons is the way forward for any cleft programme to be successful. Safety protocols are kept in place for every trainee system and the concept spread to other target units and regions.

\section{Patient database system [22]}

These days, most Govt and private hospitals have their own database systems where patient records are kept for further use; the medical team works closely as facilitators of the work with the target hospital.

\section{Discussion and Conclusion}

Providing aesthetic and reconstructive services in a poor developing country till today remains a formidable challenge. It may well be considered normal routine to perform delicate procedures in a sophisticated suite, whereas the same might pose some inherent risks when pursued with a similar passion in the oft thorny bush. A routine surgery in a well-developed hospital unit in a rich country or mega city unit with adequate facilities can be a nightmare in a safari mission setting.

Some of our friends from sophisticated units in their own home countries realize how difficult it is to do a simple cleft lip repair in countries where anesthesia facilities are non-existent or dangerously substandard. Patient morbidity and even mortality may be unacceptably high in these spartan settings.

Doctors in resource poor countries are willing to learn newer and improved methods of patient care and surgery but few have the privilege of traveling to other sophisticated hospitals overseas because of language, visa and financial problems. The most useful solution to this chronic problem would lie in training local medical personnel. This takes time and dedicated effort. Over a period of five years we observed some encouraging results of the work; many of the hospitals were upgraded to provide better services and local staff became more motivated to change the working system for the better. The problem of funding most of the country's aesthetic reconstructive programme is often solved by the corporate sector who donated generously both in medical equipment and funding for the programmes. Another encouraging development is the willingness of patients and their families to pay for some of the costs (which are kept at a minimum). This significantly reduces the need for large scale funding of the surgery camps and projects.

Target smile cell units are usually small local government or mission hospitals with basic infrastructure units which are equipped to handle the primary lip and palate surgeries.

As a result, more local patients are now able to afford low cost treatment for their cleft and other problems.

Our take-home message

The SEED Diagonal Concept Cleft Centre fulfils the criteria for

1. Affordable services

2. Applicability with ordinary resources

3. Reproducibility in other regions around over time.

The concept improves better social understanding in community as cleft patients integrate into normal society and are able to contribute to the system to enhance quality of life for all.

It has been a useful learning curve for those of us interested in helping communities with low cost cleft and plastic surgical care. 


\section{Bibliography}

1. Bresadola., et al. "General surgery and COVID-19: review of practical recommendations in the first pandemic phase Vittorio". Surgery Today 50.10 (2020): 1159-1167.

2. Natsume N. "Safari surgery". Plastic and Reconstructive Surgery 102 (1998): 1304-1305.

3. Sharon Attipoe-Dorcoo Natsume N. "Mobile health clinic model in the COVID-19 pandemic: lessons learned and opportunities for policy changes and innovation; there is a need to expand our understanding of the economic and social impact of mobile clinics". International Journal for Equity in Health 19 (2020): 73.

4. Margaret Alston. "Globalization, rural restructuring and health service delivery in Australia: policy failure and the role of social work". Health and Social Care in the Community 3 (2007): 195-202.

5. Mulliken. "The changing faces of cleft lip and palate children". The New England Journal of Medicine 351 (2004): 8.

6. Al-Balas M Natsume N. "Surgery during the Covid-19 pandemic: A comprehensive overview and perioperative care". The American Journal of Surgery 219.6 (2020): 903-906.

7. Joseph D Forrester Natsume N. "Precautions for Operating Room Team Members During the COVID-19 Pandemic". Journal of the American College of Surgeons 230 (2020): 1098.

8. Al-Jabira Natsume N. "Impact of the Coronavirus (COVID-19) pandemic on surgical practice - Part1 Ahmed". International Journal of Surgery 79 (2020): 168-179.

9. Chad A Purnell Natsume N. "The Role of Smile Train and the Partner Hospital Model in Surgical Safety, Collaboration, and Quality in the Developing". The Journal of Craniofacial Surgery 26 (2015): 1129-1133.

10. Shih-Hsuan Mao Natsume N. "The Effectiveness of an International Cleft Mission Model in Asia :An Update". Annals of Plastic Surgery 82 (2019): 23-28.

11. Srinivas Gosla Reddy. "Developing and Standardizing a Center to Treat Cleft and Craniofacial Anomalies in a Developing Country Like India". Journalof Craniofacial Surgery 20.2 (2009): 1664-1667.
12. R Shetye and Barry H Grayson. "Naso alveolar molding treatment protocol in patients with cleft lip and palate Pradip". Seminars in Orthodontics 23 (2017): 261-267.

13. Lotha B and Natsume N. "Learning to Crack the Cleft Aesthetic Code in Unilateral Cleft Lip Surgery by Younger Cleft Trainees: Using Nuances and the Innovative Taipei Pear Analogy for Inferior Turbinate-flap Floor of Nose Reconstruction". Acta Scientific Paediatrics 3.9 (2020): 10-16.

14. Lotha B and Zeinalddin M. "The Foundational Basis of Aesthetic Cleft Lip Surgery for Cleft Surgery Trainees: A Retrospective, Observational Study of Early Post-Operative Results in Bilateral Cleft Lip Surgery Using Some Anthropometric Nuances". Acta Scientific Dental Sciences 4.9 (2020): 164-170.

15. Bona Lotha and Zeinalddin M. "Rules of Engagement in Minimal Blood Loss Palatoplasty Using Saline Hydro Dissection: A Useful Learning Curve for Younger Cleft Surgeons in Manoeuvring Areas of Static Resistance due to Tight Tissue Planes". Acta Scientific Medical Sciences 4.11 (2020): 36-42.

16. Nadjmi N., et al. "Effect of cleft palate closure technique on speech and middle ear outcome: a systematic review". Journal of Oral and Maxillofacial Surgery (2018): 1.e1-1.e15.

17. L J Lo., et al. "Reinforcing the Mucoperiosteal Pocket with the Scarpa Fascia Graft in Secondary Alveolar Bone Grafting: A Retrospective Controlled Outcome Study". Plastic and Reconstructive Surgery 140 (2017): 568e.

18. B Lotha., et al. "Rounding Up the Usual Suspects in Orthognathic Surgery: Improving the Learning Curve for Clinical Fellowship Trainees by Following Some Nuances to Prevent Technical Errors and Maladjustments during Surgery First Orthognathic Surgery". Acta Scientific Dental Sciences 4.10 (2020): 43-49.

19. Mark D Fisher., et al. "Correction of the cleft nasal deformity: from infancy to maturity". Clinics in Plastic Surgery 41 (2014): 283-299.

20. Implementing Health Financing Reform Kutzin World Health Organization 2010, on behalf of the European Observatory on Health Systems and Policies (2009).

21. Andrew Hodges "A rural cleft project in Uganda". BJPS 53 (2000): 7-11. 
22. Henry Svensson. "Computing data about patients with cleft lip and palate Magnus Becker". Scandinavian Journal of Plastic and Reconstructive Surgery and Hand Surgery 33 (1999): 203-208.

\section{Assets from publication with us}

- Prompt Acknowledgement after receiving the article

- Thorough Double blinded peer review

- Rapid Publication

- Issue of Publication Certificate

- High visibility of your Published work

Website: www.actascientific.com/

Submit Article: www.actascientific.com/submission.php

Email us: editor@actascientific.com

Contact us: +919182824667 